\title{
RADIO EMISSION FROM AKARI GALAXIES
}

\author{
A. Pepiak ${ }^{1}$, A. Solarz ${ }^{2}$, A. Pollo ${ }^{1,3}$, T. T. Takeuchi ${ }^{2}$, W. Jurusik ${ }^{1}$, and AKAri Team \\ ${ }^{1}$ Astronomical Observatory of the Jagiellonian University, Kraków, Poland \\ ${ }^{2}$ Department of Particle and Astrophysical Science, Nagoya University, Japan \\ ${ }^{3}$ National Centre for Nuclear Research, Warszawa, Poland \\ E-mail: agata@oa.uj.edu.pl \\ (Received July 08, 2012; Accepted August 15, 2012)
}

\begin{abstract}
It is a long known fact that there exists a tight correlation between far-infrared and radio emission both for galaxies hosting active galactic nuclei and for star forming galaxies. We probe the radio - infrared correlation for a sample of extragalactic sources constructed by the cross-correlation of the AKARI/IRC All-Sky Survey Point Source Catalogue, the AKARI/FIS All-Sky Survey Bright Source Catalogue, and the NRAO VLA Sky Survey. Additionally, all objects of our sample were identified as galaxies in NED and SIMBAD databases, and a part of them is known to host active galactic nuclei (AGNs). After remeasuring all the fluxes, in order to avoid small aperture effects, we compare the ratio of radio to infrared emission from different types of extragalactic sources, and discuss the FIR/radio correlation as seen by AKARI and make a comparison to the previous results obtained thanks to IRAS.
\end{abstract}

Key words: infrared; radio; galaxies; FRC

\section{INTRODUCTION}

The radio and far-infrared (FIR) luminosities of galaxies are approximately linearly correlated (e.g., Helou et al., 1985; Mauch \& Sadler, 2007; Morić et al., 2010). The far-infrared - radio correlation (FRC) is one of the most universal empirical correlations known among the global parameters of observed galaxies. It spans a wide range of galaxy types and seems to be valid both for the local and the distant universe. Presently FRC is believed to be driven mostly by star formation. However, the infrared and radio emission mechanisms in star forming galaxies involve very different physical processes and timescales. Moreover, the correlation is also observed for AGN-hosting galaxies. Hence, it is not yet established if the FRC is related only to star formation activity, or maybe some other processes are involved.

This paper presents some preliminary results of studies of the FRC for nearby galaxies $(z \lesssim 0.2)$ based on far-infrared data from the AKARI All-Sky Survey and radio data from the NRAO VLA Sky Survey.

\section{SAMPLE SELECTION}

The galaxy sample (Table 1) selection was made through a multi-step proceedure. The particular steps are described below.

1. Cross-correlation of two AKARI all-sky catalogues: AKARI IRC All-Sky Survey Point Source Catalogue (mid-infrared bands: 9 and $18 \mu \mathrm{m})$ and AKARI FIS All-Sky Survey Bright Source Catalogue (FIR bands: 65, 90, 140, and $160 \mu \mathrm{m}$ ) with search radius of $15^{\prime \prime}$;

2. Constraints for extinction: maps of the Galactic cirrus emission at $100 \mu \mathrm{m}$ were used to reject sources from the Galactic plane and Magellanic Clouds (low $100 \mu \mathrm{m}$ emissivity: $\left.I_{100 \mu \mathrm{m}}<10 \frac{\mathrm{MJy}}{\mathrm{sr}}\right)$ 
TABLE 1.

Sources Statistics

\begin{tabular}{|c|c|}
\hline IRC catalogue & 870973 \\
\hline FIS catalogue & 427071 \\
\hline IRC-FIS cross-correlation & 50809 \\
\hline Galactic extinction $I_{100 \mu \mathrm{m}}<10 \frac{\mathrm{MJy}}{\mathrm{sr}}$ & 3093 \\
\hline Galaxies & 1546 \\
\hline $\begin{array}{c}\text { Galaxies whose positions are } \\
\text { available for NVSS }\left(\delta>-40^{\circ}\right)\end{array}$ & 1246 \\
\hline $\begin{array}{c}\text { Galaxies with measurable radio flux } \\
\text { density on NVSS images* }\end{array}$ & 1128 \\
\hline Galaxies with AGN activity* & 301 \\
\hline Galaxies with no known AGN activity* & 827 \\
\hline
\end{tabular}

* - with redshift information available

TABLE 2.

$q$ Parameter

\begin{tabular}{|c|c|c|}
\hline \multicolumn{1}{|c}{$q$ Parameter } \\
\hline Whole sample & $\langle q>=2.23$ & 0.44 \\
\hline AGNs & $\left\langle q_{\mathrm{AGN}}>=2.04\right.$ & 0.65 \\
\hline 'Normal' galaxies & $\left\langle q_{\mathrm{SF}}>=2.30\right.$ & 0.31 \\
\hline
\end{tabular}

3. Identification and selection of galaxies: information from NED and SIMBAD databases and optical images from Digital Sky Survey (DSS) were used to select the sample of galaxies;

4. Radio fluxes measurements: $1.4 \mathrm{GHz}$ NRAO VLA Sky Survey (NVSS) continuum total intensity images were used to carry out measurements of radio fluxes at the level of $3 \sigma$ brightness fluctuations of the images $(\sigma \sim 45 \mathrm{mJy})$;

5. Additionally: ancillary information was collected (types, morphology, redshifts and photometry in various wavelengths).

\section{PRELIMINARY RESULTS}

Our results based on AKARI FIS WIDE-L band (centered at $90 \mu \mathrm{m})$ and NVSS data are shown in Fig. 1.

The FRC can be quantified by its slope: $q=\log \left(\frac{S_{F I R}}{S_{\text {rad }}}\right)$, where $S_{F I R}$ denotes the far-infrared flux and $S_{\text {rad }}$ denotes the radio flux. Our preliminary results are presented in Table 2 . They are consistent with $\langle q\rangle \sim 2.3$ from previous studies for the local universe.

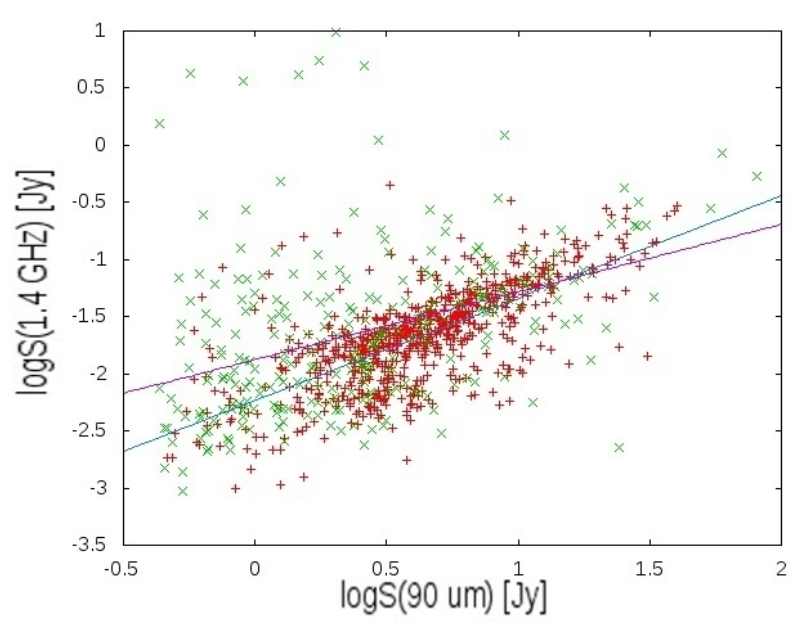

Fig. 1. Radio vs. FIR flux for our sample of AKARI nearby galaxies. Green crosses represent galaxies with AGN activity, red crosses represent galaxies with no known AGN activity ('normal' galaxies). Best fit lines: blue 'normal' galaxies, red - AGNs.

\section{SUMMARY}

We confirmed a tight FRC for AKARI local galaxies with $\langle q\rangle \approx 2.23$. We found that $\mathrm{FIR} /$ radio ratio for galaxies with AGN activity is lower than for normal galaxies. However, this ratio is still within the scatter of the correlation for galaxies with no information about AGN activity. Our results are consistent with previous results from the literature for both AGNs and 'normal' star-forming galaxies.

\section{ACKNOWLEDGEMENTS}

This work is based on observations with AKARI, a JAXA project with the participation of ESA. This research has made use of the NED and the SIMBAD databases. This reseach has been supported by the grant of the Polish National Science Centre N N203 512938 . This research was partially supported by the project POLISH-SWISS ASTRO PROJECT cofinanced by a grant from Switzerland through the Swiss Contibution to the enlarged European Union. T. T. Takuechi has been supported by the Grant-in-Aid for the Scientific Research Fund (20740105, 23340046, and 24111707) and for the Global COE Program Request 
for Fundamental Principles in the Universe: from Particles to the Solar System and the Cosmos commissioned by the Ministry of Education, Culture, Sports, Science and Technology (MEXT) of Japan.

\section{REFERENCES}

Helou, G., Soifer, B. T., Rowan-Robinson, M., 1985, Thermal Infrared and Nonthermal Radio - Remarkable Correlation in Disks of Galaxies, ApJ, 298, L7

Mauch, T. \& Sadler, E. M., 2007, Radio Sources in the 6dFGS: Local Luminosity Functions at $1.4 \mathrm{GHz}$ for Star-Forming Galaxies and Radio-Loud AGN, MNRAS, 375, 931

Morić, I., Smolčić, V., Kimball, A., Riechers, D. A., Ivezić, Ž., \& Scoville, N., 2010, A Closer View of the Radio-FIR Correlation: Disentangling the Contributions of Star Formation and Active Galactic Nucleus Activity, ApJ, 724, 779 